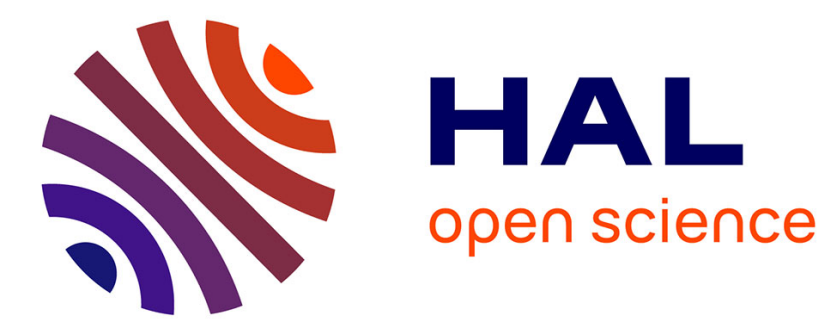

\title{
Charge-density wave phase-slip in niobium triselenide: dislocations and the growth of an, electronic crystal
} J. Gill

\section{To cite this version:}

J. Gill. Charge-density wave phase-slip in niobium triselenide: dislocations and the growth of an, electronic crystal. Journal de Physique IV Proceedings, 1993, 03 (C2), pp.C2-165-C2-170. 10.1051/jp4:1993233 . jpa-00251316

\section{HAL Id: jpa-00251316 https://hal.science/jpa-00251316}

Submitted on 1 Jan 1993

HAL is a multi-disciplinary open access archive for the deposit and dissemination of scientific research documents, whether they are published or not. The documents may come from teaching and research institutions in France or abroad, or from public or private research centers.
L'archive ouverte pluridisciplinaire HAL, est destinée au dépôt et à la diffusion de documents scientifiques de niveau recherche, publiés ou non, émanant des établissements d'enseignement et de recherche français ou étrangers, des laboratoires publics ou privés. 


\title{
Charge-density wave phase-slip in niobium triselenide: dislocations and the growth of an electronic crystal
}

\author{
J.C. GILI
}

H.H. Wills Physics Laboratory, University of Bristol, Tyndall Avenue, Bristol BS8 1TL, Great-Britain

\begin{abstract}
The analogy is explored between phase-slip in charge-density wave conductors, which brings about the growth and decay of an electronic crystal, and the processes of growth and plastic flow which occur in conventional crystals. The experimental data on $\mathrm{NbSe}_{3}$ are re-examined, and new measurements of the voltage $V_{p s}$ needed to produce phase-slip at a given rate presented. It is suggested that $V_{p s}$ is determined mainly by the difficulty of inducing dislocation loops in the charge-density wave to climb to the crystal surface, rather than by their difficulty of nucleation.
\end{abstract}

\section{Introduction.}

Niobium triselenide $\left(\mathrm{NbSe}_{3}\right)$ is one of those quasi low-dimensional metals, with conducting chains weakly coupled together, in which an effective interaction between the conduction electrons leads to their ordering, below a critical temperature $T_{P}$, into the type of electronic crystal known as a charge-density wave (CDW). The density of electrons exhibits a spatially periodic modulation whose wavevector $\mathbf{Q}$, in the absence of distortion, has component $2 \mathrm{k}_{\mathrm{F}}$ in the chain direction $\mathrm{z}$, where $\mathrm{k}_{\mathrm{F}}$ is the Fermi wavevector (1). When, as in $\mathrm{NbSe}_{3}, \mathrm{Q}$ is incommensurate with the reciprocal lattice, the energy has no inherent dependence on the phase of the CDW which, in a perfect crystal, would be induced to move by an infinitesimal electric field along $z$. Such a collective motion of the entire electronic distribution leads to the co-operative conduction proposed by Fröhlich [1]. In real crystals the phase is "pinned" by chemical impurities and other defects, and continuous Fröhlich conduction commences only when the field E exceeds a threshold value $\mathrm{E}_{\mathrm{T}}[2]$.

A CDW is usually described by a continuous order parameter $\Delta \cos [\mathbf{Q} . \mathbf{r}+\phi(\mathbf{r}, \mathrm{t})]$, with $\Delta$ (a measure of the energy gap) constant, and phase $\phi$ single-valued. However, as steady conduction is observed between current terminals attached (in the case of $\mathrm{NbSe}_{3}$ ) to the side of the ribbon-like crystal, beyond which the CDW remains pinned, its motion requires wavefronts to be added and removed near the terminals. This happens through processes of phase-slip, in which $\Delta$ collapses locally and the continuity of $\phi$ is lost. As they effect the growth and decay of the electronic crystal, phase-slip processes are expected to resemble those of growth and plastic flow in conventional crystals [3].

Thus it is accepted that in macroscopic crystals the addition or removal of each wavelength of CDW proceeds through the formation of an edge dislocation loop (phase vortex), which then expands to the crystal surface. The factor limiting the rate of phase-slip is thought to be that at which loops are nucleated in the stressed CDW. A recent theory of the nucleation process [4] appears consistent with observations [5] (1) As its components in crystallographic directions other than the chain axis are invariably commensurate, $Q$ will be defined (by suitable choice of unit cell) to lie along that axis. 
of the relation between the rate of phase-slip, given by the Fröhlich current $\mathrm{I}_{\mathrm{C}}$, and the stress required to induce it, measured as a voltage $V_{p s}$ in excess of that needed to drive $I_{C}$ in the bulk material.

The agreement is, however, rather surprising if phase-slip is indeed analogous to growth and plastic flow in conventional crystals. In the latter, although nucleation is a possibility, new layers or dislocation loops are almost always generated, without nucleation, from existing dislocations. As similar mechanisms should be available in CDWs, it seems useful to enquire whether factors other than nucleation contribute to $\mathrm{V}_{\mathrm{ps}}$. Accordingly, processes of crystal growth and plastic flow of possible relevance to CDW phase-slip are outlined in $\S 2$. The experimental data are considered, and some new results presented, in $\S 3$. Finally, in $\$ 4$, an attempt is made to identify the important features of the phase-slip process in $\mathrm{NbSe}_{3}$.

\section{Dislocations and phase-slip in charge-density waves.}

Dislocations in CDWs, discussed by Feinberg and Friedel [6], differ in some respects from those in conventional crystals. The Burgers vector is necessarily parallel to $\mathbf{Q}$, and of magnitude $2 \pi / \mathrm{Q}$ (the CDW wavelength $\lambda$ ), so that screw dislocations must lie parallel, and edge dislocations perpendicular, to the chain axis $\mathrm{z}$. The climb of edge dislocations perpendicular to $\mathrm{z}$, which in conventional crystals requires the migration of atoms or vacancies, now involves mobile electrons, readily available in $\mathrm{NbSe}_{3}$ even at low temperatures. Nevertheless, climb is expected to be more difficult than glide parallel to $\mathrm{z}$, on account of the extreme elastic anisotropy of the CDW, a result of the weak inter-chain coupling. Clearly, to sustain phaseslip, the longitudinal stress $\Sigma$ associated with $V_{p s}$ has to be sufficient both to generate dislocation loops, and to induce their climb to the crystal surface. Mechanisms of generation are considered first.

\subsection{The generation of dislocation loops.}

(a) Nucleation processes.

For an edge dislocation loop to grow indefinitely in a CDW, it must first reach a critical size dependent on the stress $\Sigma$. A process of nucleation is required if the $\mathrm{CDW}$ is initially free of dislocations. Ramakrishna et al. [4], in their theory of thermal nucleation, give the critical radius (omitting a numerical factor $\sim 1$ ) as

$$
\mathrm{r}_{\mathrm{c}} \approx \mathrm{K} / 2 \mathrm{Q} \Sigma
$$

where $\mathrm{K}$ is an effective elastic modulus for the CDW. From the energy ( $\approx(\pi K)^{2 /\left(2 \Sigma Q^{3}\right)}$ ) of such loops is estimated their rate of generation and, assuming each climbs directly to the crystal surface (advancing the CDW by $\lambda$ ), the Frohlich current $I_{C}$ sustained by $V_{\text {ps. }}$. For a CDW carrying charge density $\rho$, stressed by a uniform field $V_{p s} / L$ between terminals $L$ apart (so that $\Sigma$ varies linearly between values $\pm \rho . V p s / 2$ at the terminals), the current at temperature $T$ is

$$
\mathrm{I}_{\mathrm{C}} \quad=\quad \mathrm{I}_{0}\left(\mathrm{~V}_{\mathrm{ps}} / \mathrm{V}_{\mathrm{a}}\right) \exp \left(-\mathrm{V}_{\mathbf{a}} / \mathrm{V}_{\mathrm{ps}}\right)
$$

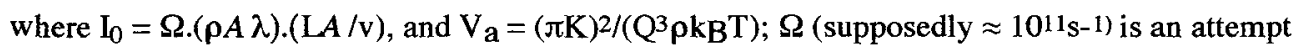
frequency, $\rho A \lambda$ ( $A$ beingthe cross-sectional area of the crystal) is the charge conveyed per phase-slip event, and $L A / v$ is the number of independent regions, of the coherence volume $v=\xi_{x} \xi_{y} \xi_{z}$, in which events may commence. A factor $V_{p s} / V_{a}$ enters because the events are concentrated in regions near the terminals whose combined length is effectively $\mathrm{L}_{\mathrm{ps}}=\mathrm{L} \cdot \mathrm{V}_{\mathrm{ps}} / \mathrm{V}_{\mathrm{a}}$.

For the CDW in $\mathrm{NbSe}_{3}$ with $\mathrm{T}_{\mathrm{P}}=144 \mathrm{~K}$ (the subject of $\S 3$ ), $\mathrm{Q}=4.5 \times 10^{7} \mathrm{~cm}^{-1}, \rho(\mathrm{T}=0) \approx 300 \mathrm{C} . \mathrm{cm}^{-3}$ $\left(\mathrm{n}=1.9 \times 10^{21}\right.$ electrons $\left.\mathrm{cm}^{-3}\right)$, and $\mathrm{v} \approx 70 \AA^{3}$ [7]. For an edge dislocation loop $\mathrm{K}=\left(\mathrm{K}_{\mathrm{ZZ}} \mathrm{K}_{\perp}\right)^{1 / 2}$, where $\mathrm{K}_{\perp}$ is the geometric mean of the shear moduli $\mathrm{K}_{\mathrm{xz}}, \mathrm{K}_{\mathrm{yz}}$. With a mean-field value $2 \mathrm{nE} \mathrm{F}_{\mathrm{F}} \approx 110 \mathrm{~J} . \mathrm{cm}-3$ for $\mathrm{K}_{\mathrm{ZZ}}$ (with Fermi energy $\mathrm{E}_{\mathrm{F}}$ based on the free-electron mass), and $\mathrm{K}_{\mathrm{Zz}}: \mathrm{K}_{\mathrm{Xz}}: \mathrm{K}_{\mathrm{yz}}=1: 1 / 81: 1 / 625$ (from the squares of the coherence lengths $\xi_{\mathrm{b}} *: \xi_{\mathrm{a}} *: \xi_{\mathrm{c}} *=1: 1 / 9: 1 / 25$, derived from measured Lee-Rice phase correlation length $\left\{\right.$ [7], and letting $x, y, z$ be the crystallographic directions $\left.\mathrm{a}^{*}, \mathrm{c}^{*}, \mathrm{~b}^{*}\right), \mathrm{K} \approx 7.3 \mathrm{~J} . \mathrm{cm}^{-3}$ 

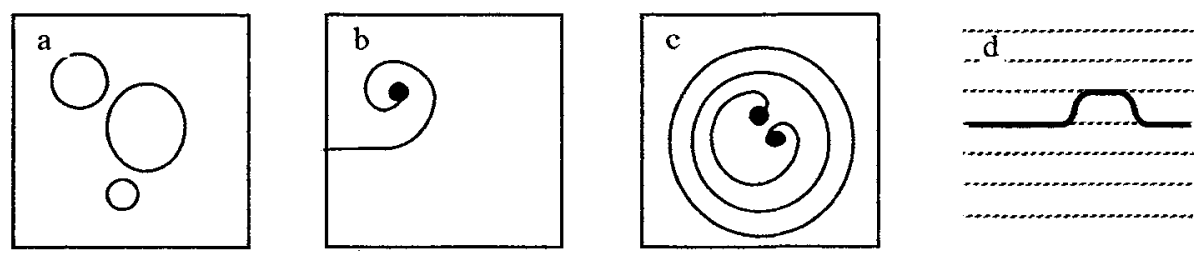

Figure 1. Illustrating edge dislocations near a terminal (a), nucleated thermally; (b) and (c), associated with Frank-Read structures; and (d), climbing by forming kinks. In (b) and (c), screw dislocations ( $\bullet$ ) extend to the opposite terminal. The chain direction $\mathrm{z}$ is normal to the paper.

when $\mathrm{T}=0$. From these one obtains the low-temperature values $\mathrm{V}_{\mathrm{a}} \approx 1400 / \mathrm{T} \mathrm{mV} . \mathrm{K} ; \mathrm{r}_{\mathrm{c}} \approx 55 / \mathrm{V}_{\mathrm{ps}} \AA . \mathrm{mV}$ near the terminals; and, for a crystal of dimensions $\mathrm{L}=1 \mathrm{~mm}$ and $A=10 \mu \mathrm{m}^{2}, \mathrm{I}_{0} \approx 6 \times 10^{11} \mathrm{~A}$.

Though the above model of nucleation is the more relevant here, it is mentioned that a fundamentally different process has been suggested by Brazovskii and Matveenko [8]. Rather than being generated by $\mathrm{V}_{\mathrm{ps}}$, the dislocations appear spontaneously, in order to screen the field associated with the contact potential between the $\mathrm{CDW}$ material and the metal of the terminal. However, in $\mathrm{NbSe}_{3}$, which remains metallic at $\mathrm{T}=0$, the screening is already provided by conduction electrons not concerned in the CDW.

\section{(b). Generation without nucleation.}

As with slip in a conventional crystal, unlimited phase-slip in a CDW should be possible without any need for dislocation loops to be nucleated, if certain structures suggested by Frank and Read [9] are initially present. The processes differ, however, as the restriction of the Burgers vector in CDWs makes impossible the glide of dislocations in two dimensions which leads to slip in conventional crystals. The equivalent processes in CDWs, analogues of those leading to crystal growth, involve climb in two dimensions.

In the simplest Frank-Read (FR) structure (figure 1b), a screw dislocation is joined to the crystal surface by edge sections near each terminal. Continuous phase-slip merely requires the edge sections (which assume a spiral form) to climb under the influence of applied stress. Such structures are perhaps of importance near the crystal surface; elsewhere screw dislocations of opposite senses, presumably of similar abundance, will link to form FR sources for the multiplication of dislocation loops.

Such a source, consisting of a mixed dislocation loop in which opposite screw sections are joined near each terminal by edge sections, is shown in figure $1 \mathrm{c}$. Climb induced by longitudinal stress causes each edge section to curve, eventually releasing a dislocation loop which continues to expand, after which the process repeats. The critical stress for the process to occur is (cf. expression 1)

$$
\Sigma_{\mathrm{c}}=\mathrm{K} / \mathrm{Qd}
$$

where $d$ is the separation of the screw sections.

The abundance of such structures in CDWs is difficult to estimate. There is no energetic objection to their presence in equilibrium: dislocations should be stable at separations not much greater than the Lee-Rice correlation length, their elastic energy being then of the same order as that already associated with the weak pinning. During Fröhlich conduction the drift with the CDW of loops initially in the bulk material will result in their being eliminated at the positive terminal, after being temporarily active as FR sources. Mixed loops initially ending near the negative terminal will, however, persist: their activity as FR sources creating new CDW compensates for the drift, so that the loops extend and eventually provide FR sources for the removal of CDW at the positive terminal. When current flows it is likely that further such loops will appear, as a result of non-uniform shear, where terminals make contact over only part of the crystal width. 
It would not be surprising, therefore, were the number of FR sources to approach, in order of magnitude, that of the Lee-Rice "domains" in the cross-section $A$. With $d$ then of the same order as a transverse Lee-Rice length, typically $1 \mu \mathrm{m}$, and other quantities as previously assumed, the critical stress $\Sigma_{\mathbf{c}}$ is reached with $\mathrm{V}_{\mathrm{ps}} \approx 10 \mu \mathrm{V}$, about two orders of magnitude smaller than is needed to induce appreciable phase-slip by nucleation. FR sources should then dominate the generation of dislocation loops.

\subsection{Expansion to the crystal surface.}

The stress-induced climb of the loops to the crystal surface completes the phase-slip process (glide, induced by shear stress is a likely accompaniment, and extends the region where phase-slip occurs). Although its importance is recognised [6], no quantitative treatment of the effect of the lattice in impeding the climb of CDW dislocations is available. In conventional crystals the analogous resistance to glide is modelled [10] by the motion of a line under tension across a sinusoidal Peierls-Nabarro potential (figure $1 \mathrm{~d}$ ). When $T=0$, motion from one valley of the potential to the next requires a critical stress $\Sigma \mathrm{p}=2 \mathrm{~W} / \mathrm{b} l$, where $l$ is the distance between valleys, $b$ the Burgers vector, and $W_{P}$ the amplitude of the potential. If $T>0$ pairs of kinks can be generated thermally, with activation energy $W_{k} \approx l\left(W_{0} W_{P}\right)^{1 / 2}$, where $W_{0}$ is the energy per unit length of the dislocation; motion can then be induced by the small stress needed to dissociate the pairs.

With CDWs, $l$ becomes the chain separation, here $\approx 5 \AA$ along $\mathrm{x}\left(\mathrm{a}^{*}\right), 15 \AA$ along $\mathrm{y}\left(\mathrm{c}^{*}\right)$. With $\xi_{\mathrm{x}} \approx 3 \AA$ and $\xi_{y} \approx 1 \AA$ both smaller than the corresponding $l$, transfer between valleys, and kink motion along them, are both resisted by the discreteness of the lattice. An upper limit for $2 W_{P}$ is set by the energy $(\approx \Delta$ per chain affected) needed to collapse the $\mathrm{CDW}$, equivalent to $\mathrm{V}_{\mathrm{ps}}=\Delta \mathrm{e}, \approx 22 \mathrm{mV}$ in the mean-field case. With this value for $2 W_{\mathrm{P}}$, and $W_{0} \approx K \lambda^{2} / 2 \pi$, the activation temperature for kink pairs is about $300 \mathrm{~K}$.

\section{Experiment.}

The relation between the rate of phase-slip, measured as the Fröhlich current $\mathrm{I}_{\mathrm{c}}$, and the excess voltage $\mathrm{V}_{\mathrm{ps}}$ needed to produce it is studied by comparing the voltage-current $(\mathrm{V}-\mathrm{I})$ characteristics using different arrangements of terminals. Conventionally [5,11], comparison has been between a "normal" 4-terminal configuration $N$, in which I flows between the outer pair of terminals (separation $L_{N}$ ), and $V\left(\right.$ denoted $V_{N}$ ) is measured between the inner, and a "transposed" version $T$, with I flowing between the inner terminals (separation $L_{T}$ ), and $V_{T}$ measured between the outer. In each case the Fröhlich component $I_{C}$ of $I$ is identified with its non-linear part $I-V / R$, where $R=V / I$ measured in the linear regime with $I$ less than the threshold value $I_{T}$. For given $I_{C}, V_{T}$ is (usually) greater than $V_{N}$, as in figure 2 . The difference $V_{T N}=V_{T}-V_{N}$ clearly arises because the phase-slip regions contribute directly to $V_{T}$, but not to $V_{N}$. As $V_{p s}$ appears in $V_{T}$, but contributes only $V_{p s} \cdot L_{N} / L_{T}$ to $V_{N}$, it is usually identified with $V_{T N} /\left[1-L_{T} / L_{N}\right]$, which quantity is denoted $\mathrm{V}_{\mathrm{TN}}$ (below.

In their recent experiments on phase-slip, Maher et al [5] found $V_{T N} *$ to be related to $I_{C}$ essentially as is $\mathrm{V}_{\mathrm{ps}}$ predicted (expression 2) by the nucleation model of Ramakrishna et al [4]. However, the assumption that $\mathrm{V}_{\mathrm{ps}}$ is the only contributor to $\mathrm{V}_{\mathrm{TN}}{ }^{*}$ has been challenged [12] on the grounds that if (as the nucleation model predicts) phase-slip occurs over a finite total length $L_{p s}$, then $V_{T}$ (and hence $V_{T N^{*}}$ ) is influenced by the decrease of $I_{C}$ near each terminal. As the measured $I_{C}$ is a mean over $L_{T}$, a rate of phase-slip corresponding to a given $\mathrm{I}_{\mathrm{C}}$ in the $N$ configuration will in $T$ be measured as $\mathrm{I}_{\mathrm{C}}\left[1-\mathrm{L}_{\mathrm{ps}} / \mathrm{L}_{\mathrm{T}}\right]$. Since $\mathrm{V}_{\mathrm{T}}$ and $\mathrm{V}_{\mathrm{N}}$ refer to the same measured $\mathrm{I}_{\mathrm{C}}, \mathrm{V}_{\mathrm{TN}}{ }^{*}$ exceeds $\mathrm{V}_{\mathrm{ps}}$, approximately by $\mathrm{L}_{\mathrm{ps}} / \mathrm{L}_{\mathrm{T}} \cdot \mathrm{dV} \mathrm{N} / \mathrm{d}\left(\mathrm{InI}_{\mathrm{C}}\right) /\left[1-\mathrm{L}_{\mathrm{T}} / \mathrm{L}_{\mathrm{N}}\right]$. Comparisons of $\mathrm{V}_{\mathrm{N}}$ with the voltage $\mathrm{V}_{\mathrm{M}}$ in a "mixed" configuration $M$, with alternate current and voltage terminals, revealed values of $\mathrm{L}_{\mathrm{ps}}$ which, if they applied also in $T$, would have accounted for $85 \%$ of $\mathrm{V}_{\mathrm{TN}}$.

The use of a pair of voltage terminals near the centre of $L_{T}$, between the two phase-slip regions, allows $I_{C}$ to be measured free from this effect of $L_{p s}$. If, for given $I_{C}$, the voltages between the central terminals (separation $\mathrm{L}_{\mathrm{C}}$ ) are respectively $\mathrm{V}_{\mathrm{TC}}$ and $\mathrm{V}_{\mathrm{NC}}$ when current flows as in $T$ and $N$ respectively, then 


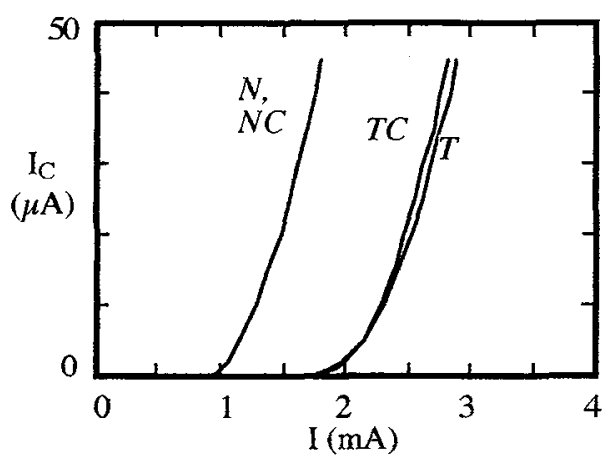

Figure 2. Fröhlich current $\mathrm{I}_{\mathrm{C}}$ vs. total current $\mathrm{I}$, recorded at $77 \mathrm{~K}$ using terminal configurations $N, T$ and versions $N C, T C$ in which voltage is measured using a central pair of terminals.

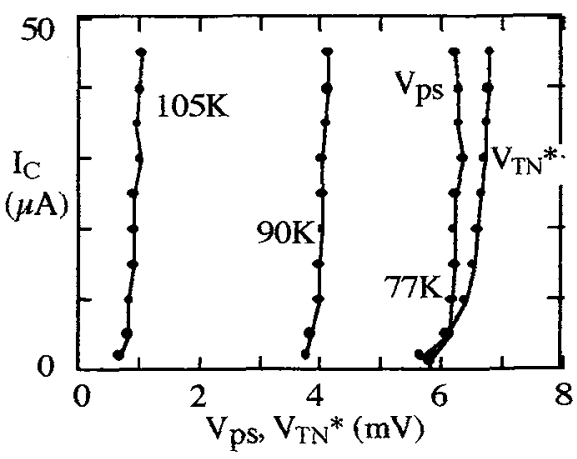

Figure 3. Phase-slip voltage $\mathrm{V}_{\mathrm{ps}}$, vs Fröhlich current $\mathrm{I}_{\mathrm{C}}$, at $77 \mathrm{~K}, 90 \mathrm{~K}$ and $105 \mathrm{~K}$. The voltage $\mathrm{V}_{\mathrm{TN}}{ }^{*}$ at $77 \mathrm{~K}$ is also shown.

$\mathrm{V}_{\mathrm{ps}}=\left(\mathrm{V}_{\mathrm{TC}}-\mathrm{V}_{\mathrm{NC}}\right) \cdot\left(\mathrm{L}_{\mathrm{T}} / \mathrm{L}_{\mathrm{C}}\right) /\left[1-\mathrm{L}_{\mathrm{T}} / \mathrm{L}_{\mathrm{N}}\right]$. In the $T$ configuration $\mathrm{Lps}_{\mathrm{ps}} / \mathrm{L}_{\mathrm{T}}=1-\mathrm{I}_{\mathrm{C}}(T C) / \mathrm{L}_{\mathrm{C}}(T)$, where $\mathrm{I}_{\mathrm{C}}(T C)$ and $\mathrm{I}_{\mathrm{C}}(T)$ are the $\mathrm{I}_{\mathrm{C}}$ measured, for same total current $\mathrm{I}$, using respectively the central and outer voltage terminals.

Measurements of $\mathrm{V}_{\mathrm{ps}}$ and $\mathrm{L}_{\mathrm{ps}}$ in $\mathrm{NbSe}_{3}$ made in this way are briefly reported here. Four crystals, with cross-sectional areas $10-100 \mu \mathrm{m}^{2}$, have been studied with similar results. The crystals (from R.E. Thorne, of Cornell University, whose help is gratefully acknowledged) had $R(300 \mathrm{~K}) / \mathrm{R}(4.2 \mathrm{~K}) \approx 250$, and $\mathrm{E}_{\mathrm{T}}(77 \mathrm{~K}) \approx 60 \mathrm{mV} . \mathrm{cm}^{-1}$. Terminals were usually of indium, about $20 \mu \mathrm{m}$ wide. As their resistances $(\sim 10 \Omega)$ indicated that the contact area was small, their perturbing effect as voltage terminals is thought to have been negligible (inner voltage terminals of gold wire, diameter $10 \mu \mathrm{m}$, resting on the crystal and presumed nonperturbing, gave similar results). Measurements of $I_{C}$ were made using a pulsed technique, with digital recording; to avoid transients associated with the rearrangement of the CDW, recording began a few ms into the pulse, and a small correction was made for the temperature rise $(\sim 50 \mathrm{mK})$ that had occurred.

The accuracy of $\mathrm{V}_{\mathrm{TN}}{ }^{*}$ as a measure of $\mathrm{V}_{\mathrm{ps}}$ may be seen from the representative records in figure 2 (obtained at $77 \mathrm{~K}$ from a crystal having $\mathrm{L}_{\mathrm{N}}=7.5 \mathrm{~mm}, \mathrm{~L}_{\mathrm{T}}=0.75 \mathrm{~mm}, A=97 \mu \mathrm{m}^{2}$ ). In this case $\mathrm{L}_{\mathrm{ps}} \approx$ $0.15 \mathrm{~L}_{\mathrm{T}}$, and $\mathrm{V}_{\mathrm{TN}} *$ exceeds $\mathrm{V}_{\mathrm{ps}}$ by less than $10 \%$. The effect of $\mathrm{L}_{\mathrm{ps}}$ thus is smaller than the measurements using the $M$ configuration suggested: the main reason is that $L_{p s}$, which clearly cannot exceed the separation $L$ of the current terminals, eventually decreases as $\mathrm{L}$ is reduced. It has also been found, using the $M$ configuration with a specimen carrying many contacts, that $L$ ps varies between current terminals $(2)$. In most cases $L_{p s}$ at each terminal is $5-10 \%$ of $L$ when $L \approx 1 \mathrm{~mm}$, but negative $L_{p s}$ (phase-slip region centred beyond the terminal) has occasionally been found. It is concluded that, in the specimens examined here, $\mathrm{L}_{\mathrm{ps}}$ was greatly influenced by dislocation glide, induced by shear associated with the varied contact geometry.

The difference between $V_{T N}$ and $V_{p s}$, though small, has important consequences for the interpretation of phase-slip. This is evident from the comparison, in figure 3, between $V_{\mathrm{TN}}{ }^{*}$ and $\mathrm{V}_{\mathrm{ps}}$ at $77 \mathrm{~K}$. The data for $\mathrm{V}_{\mathrm{TN}^{*}}$, qualitatively similar to those of Maher et al. [5], fit expression 2 with $\mathrm{V}_{\mathrm{a}} \approx 100 \mathrm{mV}$ (Maher et al. found $\mathrm{V}_{\mathrm{a}} \approx 50 \mathrm{mV}$ at this temperature, but noted substantial variation). However, most of the increase of $\mathrm{V}_{\mathrm{TN}}{ }^{*}$ with $\mathrm{I}_{\mathrm{C}}$ (and probably the variability of $\mathrm{V}_{\mathrm{a}}$ ) comes from the contribution of $\mathrm{L}_{\mathrm{ps}}$. When measured directly $V_{p s}$ is almost independent of $I_{C}:$ a fit to expression 2 gives $V_{a} \approx 450 \pm 100 \mathrm{mV}$. With increase in temperature $V_{p s}$, while remaining almost independent of $I_{C}$, decreases rapidly (from $6.2 \mathrm{mV}$ at $77 \mathrm{~K}$ to less than $1 \mathrm{mV}$ at $110 \mathrm{~K})$. Some variation $( \pm 30 \%$ at $80 \mathrm{~K})$ of $\mathrm{V}_{\mathrm{ps}}$ between specimens has also been observed.

(2) Lps is also influenced if I is diverted through voltage terminals (R.E. Thome, private communication). 


\section{Discussion.}

Although larger than the estimate in $\S 2(18 \mathrm{mV}$ at $77 \mathrm{~K})$, a value $\mathrm{V}_{\mathrm{a}} \approx 450 \mathrm{mV}$ is not in itself inconsistent with the nucleation model. $\mathrm{X}$-ray measurements [13] of the strain in the CDW associated with $\mathrm{V}_{\mathrm{TN}}{ }^{*}(\approx \mathrm{Vps})$ indicate that $\mathrm{K}_{\mathrm{ZZ}}$ is about 5 times the mean-field value (a similar estimate has been obtained from the charge transferred by the transient $I_{C}$ seen on reversing $I$, with $I>I_{T}$ but insufficient to overcome $V_{p s}$ ). This leads, as $V_{a}$ is proportional to $K_{z z}{ }^{2}$, to $V_{a} \approx 450 \mathrm{mV}$. However, despite the apparent agreement with experiment, nucleation with such large $V_{a}$ would require $V_{p s}$ to be much greater than is observed: the apparent value $\mathrm{V}_{\mathrm{a}} / \mathrm{V}_{\mathrm{ps}} \approx 75$, with $\Omega$ and $\mathrm{v}$ as before, gives a rate of phase-slip events $\sim 10^{-9} \mathrm{~s}^{-1}$, or about $10^{-15}$ of that corresponding to the measured $I_{C}$. It seems, therefore, as the analogy with crystal growth leads one to expect, that the nucleation of dislocation loops plays a negligible part in phase-slip in $\mathrm{NbSe}_{3}$.

It is concluded that the loops are generated from existing dislocations, which act as FR sources (for which, in very small $\mathrm{NbSe}_{3}$ specimens, there is already possible evidence [14]). As the stress necessary to activate FR sources in the present crystals can hardly account for more than a few tens of $\mu \mathrm{V}$, most of $\mathrm{V}_{\mathrm{ps}}$ must be attributed to the climb which completes the phase-slip process.

However, a quantitative explanation of the magnitude and behaviour of $V_{p s}$ remains elusive. The estimates in $\$ 2.2$ suggest that thermally generated kinks will sustain the observed rates of phase-slip $\left(\sim 10^{6} \mathrm{~s}^{-1}\right)$, with little assistance from $\mathrm{V}_{\mathrm{ps}}$, when $\mathrm{T} \geq 77 \mathrm{~K}$. As climb across the crystal width $\mathrm{w}$ requires $w / l$ kink pairs to be generated, with activation temperature $T_{k}$, and each may appear at $\sim t / l$ independent sites in the crystal thickness $t$, a phase-slip rate $\mathrm{rps}_{\mathrm{ps}} \sim \mathrm{N} \Omega .(\mathrm{t} / \mathrm{w}) . \exp \left(-\mathrm{T}_{\mathrm{K}} / \mathrm{T}\right)$ is supported when $\mathrm{N}$ dislocations are present. Taking $\Omega=10^{1} 1_{\mathrm{s}}-1, \mathrm{~T}_{\mathrm{k}}=300 \mathrm{~K}, \mathrm{Nt} / \mathrm{w}=1$, and $\mathrm{T}=77 \mathrm{~K}$, one has $\mathrm{r}_{\mathrm{ps}}=2 \times 10_{\mathrm{s}}-1$. If, with $\mathrm{K}_{\mathrm{ZZ}}$ larger than in $\S 2, \mathrm{~T}_{\mathrm{k}}$ is such that thermal generation is suppressed, $\mathrm{V}_{\mathrm{ps}}$ becomes a threshold for climb. Its magnitude and near-independence of $\mathrm{I}_{\mathrm{C}}$ are then explicable, but not its rapid decrease with temperature. While no firm conclusion can be reached here, one possibility deserving investigation will be mentioned: namely that the mutual repulsion of dislocations from a FR source causes them to form a regular array which behaves co-operatively, so that $V_{p s}$ is the threshold for the climb of the array as a whole.

\section{References.}

[1]. Fröhlich, H., Proc. Roy. Soc. London A 223 (1954) 296.

[2]. For a review of CDW conduction, see Monceau, P. in Electronic Properties of Quasi-One-Dimensional Materials, ed. P. Monceau (Reidel, Dordrecht 1985), part II, p.139.

[3]. See, e.g., Cottrell A.H., Dislocations and Plastic Flow in Crystals (Oxford University Press, 1953).

[4]. Ramakrishna S., Maher M.P., Ambegaokar V. and Eckern U., Phys. Rev. Lett. 68 (1992) 2066.

[5]. Maher M.P., Adelman T.L., McCarten J.P., DiCarlo D.A. and Thorne R.E., Phys. Rev. Lett. 68 (1992) 3084.

[6]. Feinberg D. and Friedel J., J. Phys. Paris 49 (1988) 485; and in Low-Dimensional Electronic

Properties of Molybdenum Bronzes and Oxides, ed. C. Schlenker (Kluwer, Dordrecht 1989), p.407.

[7]. McCarten J., DiCarlo D.A., Maher M.P., Adelman T.L. and Thorne R.E., Phys. Rev. B 46 (1992) 4456.

[8]. Brazovskii S.A. and Matveenko S.I., Sov. Phys. JETP (U.S.A.) 74 (1992) 864.

[9]. Frank F.C. and Read W.T., Phys. Rev. 79 (1950) 722.

[10]. See, e.g, Ritchie I.G. and Fantozzi G., Dislocations in Solids (Elsevier, Amsterdam) 9 (1992) 57.

[11]. Gill J.C., J. Phys. C 19 (1986) 6589.

[12]. Gill J.C., Phys. Rev. Lett. 70 (1993) 331.

[13]. DiCarlo D.A., Sweetland E., Sutton M., Brock J.D. and Thorne R.E., Phys. Rev. Lett. 70 (1993) 845.

[14]. Gill J.C., Synthetic Metals 41-43 (1991) 3917. 